Copeland, B. R., \& McConnell, H. M. (1980) Biochim. Biophys. Acta 599, 95.

Cortijo, M., \& Chapman, D. (1981) FEBS Lett. 131, 245.

Crilly, J. F., \& Earnshaw, J. C. (1983) Biophys. J. 41, 211.

Cullis, P. R. (1976) FEBS Lett. 70, 223.

Demel, R. A., \& De Kruijff, B. (1976) Biochim. Biophys. Acta 457, 109.

Estep, T. N., Mountcastle, D. B., Bittonen, R. L., \& Thompson, T. E. (1978) Biochemistry 17, 1984.

Eyring, G., \& Fayer, M. D. (1984) J. Chem. Phys. 81, 4314. Eyring, G., \& Fayer, M. D. (1985) Biophys. J. 47, 37.

Fayer, M. D. (1982) Annu. Rev. Phys. Chem. 33, 63.

Fayer, M. D. (1984) IEEE J. Quantum Electron. (in press).

Forster, D., Lubensky, T. C., Martin, P. C., Swoft, J., \& Pershan, P. S. (1971) Phys. Rev. Lett. 26, 1016.

Gamble, R. C., \& Schimmel, P. R. (1978) Proc. Natl. Acad. Sci. U.S.A. 75, 3011.

Gershfeld, N. L. (1978) Biophys. J. 22, 469.

Hui, S. W., \& He, N. B. (1983) Biochemistry 22, 1159.

Jain, M. K. (1975) Curr. Top. Membr. Transp. 6, 1.

Knoll, W., Schmidt, G., Ibel, K., \& Sackmann, E. (1985) Biochemistry 24, 5240 .

Lee, A. G. (1975) Prog. Biophys. Mol. Biol. 29, 3.

Lentz, B. R., Barrow, D. A., \& Hoechli, M. (1980) Biochemistry 19, 1943.

LePesant, J. P., Powers, L., \& Pershan, P. S. (1978) Proc. Natl. Acad. Sci. U.S.A. 75, 1792.

Mabrey, S., Mateo, P. L., \& Sturtevant, J. M. (1978) Biochemistry 17, 2464.

Martin, P. C., Parodi, O., \& Pershan, P. S. (1972) Phys. Rev. A 6, 2401.

Maynard, V. (1985) Chem. Phys. Lett. 37, 1.
Mitaku, S., Ikegami, A., \& Sakanishi, A. (1978) Biophys. Chem. 8, 295.

Nelson, K. A. (1982) J. Appl. Phys. 53, 6060.

Nelson, K. A., Casalegno, R., Miller, R. J. D., \& Fayer, M. D. (1982) J. Chem. Phys. 77, 1144.

Papahadjopoulos, D., Jacobson, K., Nir, S., \& Isac, T. (1973) Biochim. Biophys. Acta 311, 330.

Phillips, M. C. (1972) Prog. Surf. Membr. Sci. 5, 139.

Powers, L., \& Clark, N. A. (1975) Proc. Natl. Acad. Sci. U.S.A. 72, 840 .

Presti, F., Pace, R. J., \& Chan, S. I. (1982) Biochemistry 21, 3831.

Recktenwald, D. J., \& McConnell, H. M. (1981) Biochemistry $20,4505$.

Sakanishi, A., Mitaku, S., \& Ikegami, A. (1979) Biochemistry $18,2636$.

Schreier, S., Polnasek, C. F., \& Smith, I. C. P. (1978) Biochim. Biophys. Acta 515, 375.

Shimshick, E. J., \& McConnell, H. M. (1973) Biochem. Biophys. Res. Commun. 53, 446.

Shinitzky, M., \& Barenholz, Y. (1978) Biochim. Biophys. Acta 515, 367.

Shinitzky, M., Skornick, E., Gorelik, E., \& Sindelar, W. (1983a) Prog. Clin. Biol. Res. 132, 425.

Shinitzky, M., Lyte, M., Heron, D. S., \& Samuel, D. (1983b) Mod. Aging Res. 3B, 175.

Tilcock, C. P. S., Bally, M. B., Farren, S. B., \& Cullis, P. R. (1982) Biochemistry 21, 4596.

Vanderkooi, J. M. (1974) Biochemistry 13, 4000.

Zlattis, A., Zak, B., \& Boyle, A. J. (1953) J. Lab. Clin. Med. $41,486$.

\title{
Effect of Short-Chain Primary Alcohols on Fluidity and Activity of Sarcoplasmic Reticulum Membranes ${ }^{\dagger}$
}

\author{
Leonor M. Almeida, Winchil L. C. Vaz, ${ }^{\ddagger}$ Jürgen Stümpel, ${ }^{\ddagger}$ and Vitor M. C. Madeira* \\ Centro de Biologia Celular, Departamento de Zoologia, Universidade de Coimbra, 3049 Coimbra Codex, Portugal \\ Received December 19, 1985; Revised Manuscript Received March 20, 1986
}

\begin{abstract}
Intramolecular excimer formation with the fluorescent probe 1,3-di(1-pyrenyl)propane, differential scanning calorimetry, and X-ray diffraction were used to assess the effect of ethanol, 1-butanol, and 1-hexanol on the bilayer organization in model membranes, sarcoplasmic reticulum (SR) lipids and native SR membranes. These alcohols have fluidizing effects on membranes and lower the main transition temperature of dimyristoylphosphatidylcholine (DMPC), but only 1-hexanol alters the cooperativity of the phase transition and significantly increases the thickness of DMPC bilayers. The interaction of the three alcohols with the SR Ca ${ }^{2+}$ pump was also investigated. Hydrolysis of ATP and coupled $\mathrm{Ca}^{2+}$ uptake are differently sensitive to the three alcohols. Whereas ethanol and 1-butanol inhibited the $\mathrm{Ca}^{2+}$ uptake, 1-hexanol stimulated it. Nevertheless, the energetic efficiency of the pump $\left(\mathrm{Ca}^{2+}\right.$ ATP) is not significantly affected by ethanol or 1-hexanol, but uncoupling was observed with 1-butanol at high concentrations. The different effects of alcohols on the activity of SR membranes rule out an unitary mechanism of action on the basis of fluidity changes induced in the lipid bilayer. Depending on the chain length, the alcohols interact with the SR membranes in different domains, perturbing differently the $\mathrm{Ca}^{2+}$-pump activity.
\end{abstract}

$\mathbf{S}$ structurat

\footnotetext{
'Supported in part by the INIC, the Gulbenkian Foundation, NATO Research Grant 0538/82, and Deutsche Forschungsgemeinschaft Grant Ei78/4-2.

'Present address: Max-Planck-Institut für Biophysikalische Chemie, D-3400 Göttingen-Nikolausberg, FRG.
}

Goldstein, 1977; Stokes \& Harris, 1982; Waring et al., 1981; Swartz et al., 1974; Retig et al., 1977; Kondo \& Kasai, 1973; Garda \& Brenner, 1984).

Traditional pharmacology groups alcohols with other chemically unrelated compounds, i.e., anesthetic agents (Seeman, 1972), sharing in common the ability to perturb membrane organization (Seeman, 1972; Paterson et al., 1972; 
Jain \& Wu, 1977; Jain et al., 1975; Lenaz et al., 1976; Lee, 1976; Chin \& Goldstein, 1981; Lyon \& Goldstein, 1983; Garda \& Brenner, 1984). This ability has been related to their pharmacological action (Trudell et al., 1973a,b; Jain et al., 1975; Haydon et al., 1977; Lee, 1976; Pang et al., 1980). Although the molecular mechanisms of action of these drugs on membrane activities are not yet completely understood, the unitary model based on the perturbation of the lipid bilayer can be ruled out (Richards et al., 1978; Franks \& Lieb, 1978).

Sarcoplasmic reticulum membranes (SR) ${ }^{1}$ have been extensively characterized in their structural and functional characteristics (Weber et al., 1966; Hasselbach, 1979; Tada et al., 1978). Studies of drug effects on this simple and well-defined system are likely to contribute for a better understanding about the molecular mechanisms of membrane interaction. Effects of primary alcohols on functional activities of SR have been previously described (Kondo \& Kasai, 1973; Hara \& Kasai, 1977); yet, no data are available about their effects on membrane fluidity and on the energetic coupling of $\mathrm{Ca}^{2+}$ pumping. Tempting to further understand the alcohol effects on membrane activities, we studied the interaction of ethanol, 1-butanol, and 1-hexanol on functional parameters of SR membranes and physical modifications reflected in the lipid fluidity, ${ }^{2}$ as determined by the use of intramolecular excimer formation of Py(3)Py in membranes (Zachariasse et al., 1982; Almeida et al., 1982). In fact, it has been shown that lipids surrounding the $\mathrm{SR} \mathrm{Ca}^{2+}$-pump enzyme modulate its function through physical interactions, including membrane fluidity (Benett et al., 1980; Johannsson et al., 1981; Almeida et al., 1984). Therefore, modulation of membrane fluidity by alcohols will help the interpretation of mechanisms by which these compounds interact with membranes.

\section{Materials ANd Methods}

Sarcoplasmic reticulum vesicles were prepared from rabbit white muscles as described elsewhere (Madeira \& AntunesMadeira, 1976), except that isolation and resuspension media contained $2.5 \mathrm{mM}$ DTT and $10 \mu \mathrm{M}$ PMS.

Protein was determined by the biuret method (Gornall et al., 1949) using bovine serum albumin as standard.

Lipids of SR were extracted as previously described (Madeira \& Antunes-Madeira, 1976). The phospholpids were quantitated by measuring the amount of inorganic phosphate (Bartlett, 1959) after hydrolysis of extracts at $180^{\circ} \mathrm{C}$ in $70 \%$ $\mathrm{HClO}_{4}$ (Böttcher et al., 1961).

Liposomes were prepared by vortexing a mixture of $2.7 \mathrm{mg}$ of phospholipids in $5 \mathrm{~mL}$ of a buffer solution containing 0.1

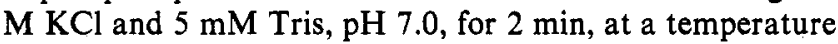
above the main phase transition of the lipid species. The mixture was then briefly sonicated in a water bath to disperse large lipid aggregates.

Incorporation of $\mathrm{Py}$ (3)Py in liposomes and SR membranes was carried out as previously described (Almeida et al., 1982, 1984). Blanks prepared under identical conditions without Py(3)Py served as controls for fluorometric measurements. These measurements were carried out with a Perkin-Elmer,

' Abbreviations: ATP, adenosine 5'-triphosphate; $\mathrm{Ca}^{2+}$-ATPase, $\left(\mathrm{Ca}^{2+}+\mathrm{Mg}^{2+}\right)$-dependent ATP-phosphohydrolase; DMPC, dimyristoylphosphatidylcholine; DPPC, dipalmitoylphosphatidylcholine; DTT, dithiothreitol; PMS, phenylmethanesulfonyl fluoride; Py(3)Py, 1,3-di(1-pyrenyl)propane; SR, sarcoplasmic reticulum; Tris, tris(hydroxymethyl)aminomethane.

2 We are using "fluidity" here in an operational sense and defining it as being directly proportional to the rate of excimer formation by the intramolecular excimer forming probe $\mathrm{Py}(3) \mathrm{Py}$. This fluidity is related to but not identical with the physical definition of fluidity.
Model MPF-3, spectrofluorometer. The excimer to monomer fluorescence intensity ratio, $I^{\prime} / I$, was evaluated as previously described (Almeida et al., 1982, 1984). This ratio changes with the fluidity of viscous media and has been used to monitor the fluidity of synthetic (Zachariasse et al., 1980) and native membranes (Melnick et al., 1981; Zachariasse et al., 1982; Almeida et al., 1982, 1984).

In order to study the effects of short-chain primary alcohols on Py(3)Py excimer formation, the alcohols were added at desired concentrations to suspensions of SR vesicles or liposomes taken from stock suspensions previously labeled with Py(3)Py; the mixtures were allowed to equilibrate with gentle stirring at room temperature for $10 \mathrm{~min}$. Indeed, a few minutes are required for the equilibrium in the sense that a constant value of $I^{\prime} / I$ is observed. It should be pointed out that the observed effects of alcohols are not related with Py(3)Py membrane/buffer partition changes, since the probe does not release into buffer even at the highest alcohol concentrations, as determined by fluorometric assay of buffer and membranes, after separation by centrifugation.

Samples for X-ray diffraction and calorimetric studies were prepared by suspending $\sim 7 \mathrm{mg}$ of lipid in $10 \mathrm{~mL}$ of $0.1 \mathrm{M}$ $\mathrm{KCl}$ and $5 \mathrm{mM}$ Tris- $\mathrm{HCl}$, $\mathrm{pH} \mathrm{7.0,} \mathrm{containing} \mathrm{the} \mathrm{desired}$ amount of primary alcohol, at a temperature $5^{\circ} \mathrm{C}$ higher than the phase transition temperature of the lipid. After equilibration for at least $3 \mathrm{~h}$ at this temperature, the suspension was centrifuged at $10000 \mathrm{~g}$ and $4^{\circ} \mathrm{C}$ for $10 \mathrm{~min}$, and the lipid pellet was directly transferred to the calorimetric pan or to the glass capillary sample tube for X-ray diffraction. The samples were immediately sealed and then subjected to differential scanning calorimetric or X-ray diffraction analysis as described by Stümpel et al. (1985).

$\mathrm{Ca}^{2+}$ uptake was monitored with a $\mathrm{Ca}^{2+}$ electrode as described elsewhere (Madeira, 1975). The reactions were carried at $20^{\circ} \mathrm{C}$ and $\mathrm{pH} 6.9$, in media $(2 \mathrm{~mL})$ containing $50 \mathrm{mM} \mathrm{KCl}$, $5 \mathrm{mM} \mathrm{MgCl}$, $10 \mathrm{mM}$ Tris-maleate, $0.27 \mathrm{mg}$ of SR protein, and $50 \mu \mathrm{M} \mathrm{CaCl}_{2}$. The uptake of $\mathrm{Ca}^{2+}$ was initiated by adding $0.2 \mathrm{mM} \mathrm{MgATP}$.

$\mathrm{Ca}^{2+}$ uptake was also estimated from the recording of ATP hydrolysis in the presence of oxalate ( $\mathrm{Ca}^{2+}$-trapping agent) as previously described (Madeira, 1982) using conditions indicated in the figure legends.

ATP hydrolysis was determined by monitoring proton production due to ATP splitting, as described previously (Madeira, 1978). Incubation media $(2 \mathrm{~mL})$ at $20^{\circ} \mathrm{C}$ contained $50 \mathrm{mM} \mathrm{KCl}, 5 \mathrm{mM} \mathrm{MgCl}_{2}, 5 \mathrm{mM}$ Tris- $\mathrm{HCl}$, $\mathrm{pH} 6.9$, $0.27 \mathrm{mg}$ of SR protein, and 0.2 or $1.0 \mathrm{mM} \mathrm{MgATP}$. The ATP hydrolysis reaction was started by adding $50 \mu \mathrm{M} \mathrm{CaCl}_{2}$. When indicated, $20 \mu \mathrm{M}$ lasalocid was added.

\section{RESUltS AND Discussion}

Effect of Short-Chain Primary Alcohols on Membrane Fluidity. (a) Model Membrane Systems. Short-chain 1alkanols perturb the bilayer organization of synthetic lipids studied by a variety of methods, namely, differential scanning calorimetry (Jain \& Wu, 1977; Sturtevant, 1982), turbidity changes (Hill, 1974; Rowe, 1983) and fluorescence (Lee, 1976). Intramolecular excimer formation of $\mathrm{Py}(3) \mathrm{Py}(\mathrm{Za}-$ chariasse et al., 1980; Almeida et al., 1984) is highly sensitive to fluidity changes and phase transitions of synthetic phospholipid bilayers. Thermotropic profiles of Py(3)Py fluorescence intensity ratio, $I^{\prime} / I$ (intramolecular excimer to monomer), are shown in Figure 1 for DMPC bilayers containing increasing concentrations of ethanol, butanol, and hexanol. In the absence of alcohols, $I^{\prime} / I$ increases with temperature, and the main phase transition of DMPC is detected as a sharp 

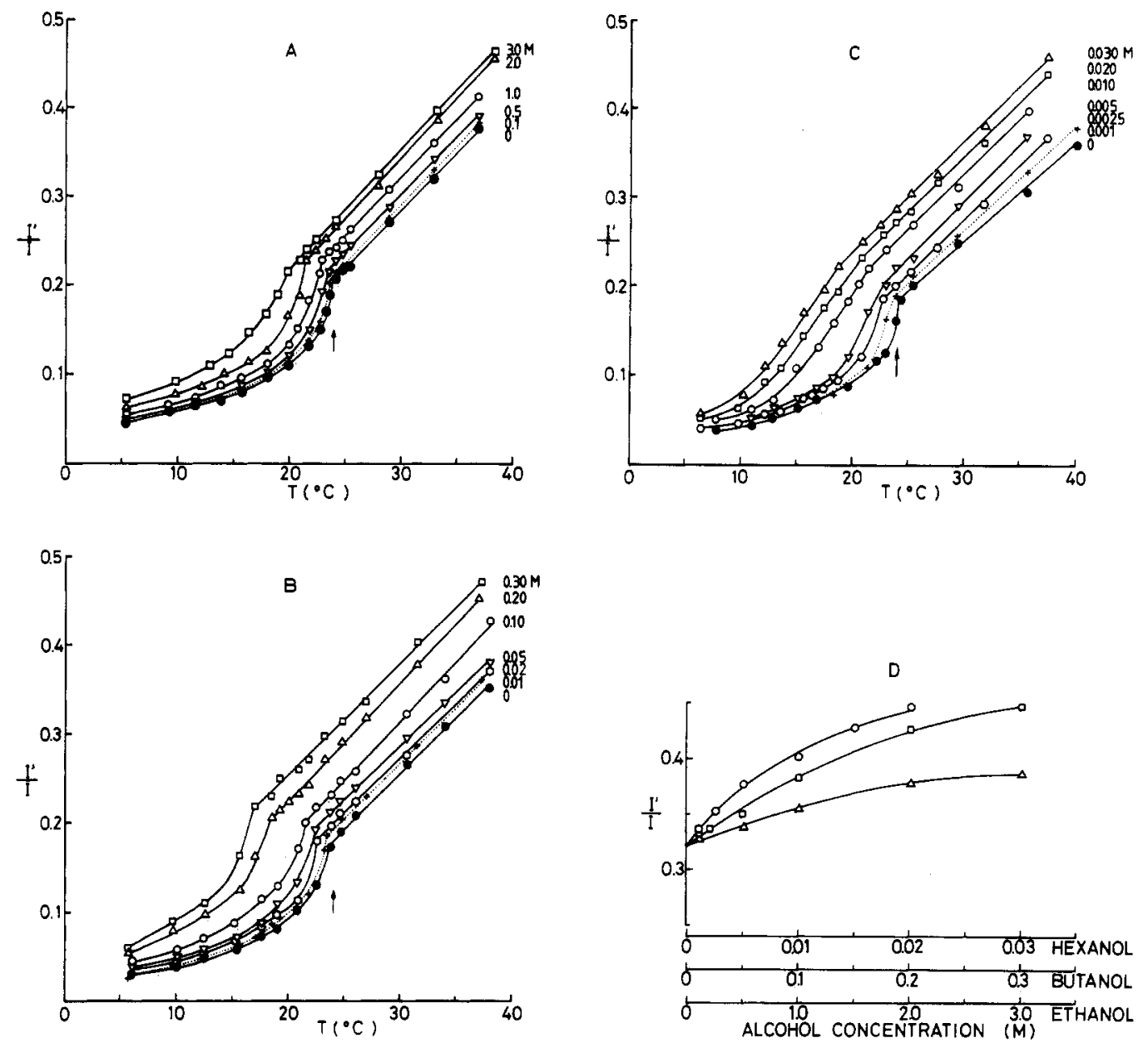

FIGURE 1: Excimer to monomer fluorescence intensity ratio, $I^{\prime} / I$, of Py(3)Py incorporated into DMPC and DMPC-alcohol mixtures, as a function of temperature. (A) Ethanol; (B) 1-butanol; (C) 1-hexanol. Alcohol concentrations (M) in lipid suspensions are indicated for each curve. The upward arrow indicates the main phase transition temperature as reported by Houslay and Stanley (1982). Values of $I^{\prime} / I$ of Py(3)Py were taken at $35^{\circ} \mathrm{C}$ from (A), (B), and (C) and were plotted, after normalization, as a function of the added alcohol concentration (D): ( $\Delta$ ) ethanol; ( $\square$ ) 1-butanol; (O) 1-hexanol. Note that the concentration ranges were deliberately chosen, e.g., 1 order of magnitude lower as the alcohol chain grows by two $\mathrm{C}$ atoms to compensate for the membrane partitions which, accordingly, increase by a factor of 10 .

Table I: X-ray Diffraction Results on the Influence of Primary Alcohols on the Structure of DMPC Multilamellar Liposomes ${ }^{a}$

\begin{tabular}{lccc}
\hline \multicolumn{1}{c}{ additive } & temp $\left({ }^{\circ} \mathrm{C}\right)$ & short sp $(\AA)$ & long sp $(\AA)$ \\
\hline none & 4 & $4.03 ; 4.12$ & 58.7 \\
2.0 M ethanol & 4 & 4.10 & 61.4 \\
0.20 M 1-butanol & 4 & 4.06 & 62.4 \\
0.02 M 1-hexanol & 4 & 4.08 & 71.0 \\
none & 35 & 4.46 & 62.4 \\
2.0 M ethanol & 35 & 4.49 & 64.4 \\
0.20 M 1-butanol & 35 & 4.51 & 63.0 \\
0.02 M 1-hexanol & 35 & 4.51 & 64.4 \\
\hline
\end{tabular}

${ }^{a}$ All preparations were in $0.1 \mathrm{M} \mathrm{KCl}$ and $5 \mathrm{mM}$ Tris- $\mathrm{HCl}, \mathrm{pH} 7.0$. The errors in the $\mathrm{X}$-ray diffraction results are $\pm 0.5 \AA$ in the long spacings and $\pm 0.01 \AA$ in the short spacings.

increase in excimer formation at a temperature range close to that observed with other techniques (Houslay \& Stanley, 1982). The alcohols increase the bilayer fluidity in terms of excimer/monomer fluorescence intensity ratio, over the entire temperature range, either below or above the phase transition, and shift the gel to liquid-crystalline phase transition to lower temperatures. By using differential scanning calorimetry, we obtained identical results (Figure 2), in agreement with Jain and Wu (1977), for DPPC. Also, similar findings have been reported by means of light scattering (Hill, 1974; Rowe, 1983) and chlorophyll $a$ fluorophore (Lee, 1976) measurements.

$\mathrm{X}$-ray diffraction studies on DMPC multilamellar liposomes were undertaken in the absence and presence of primary al-

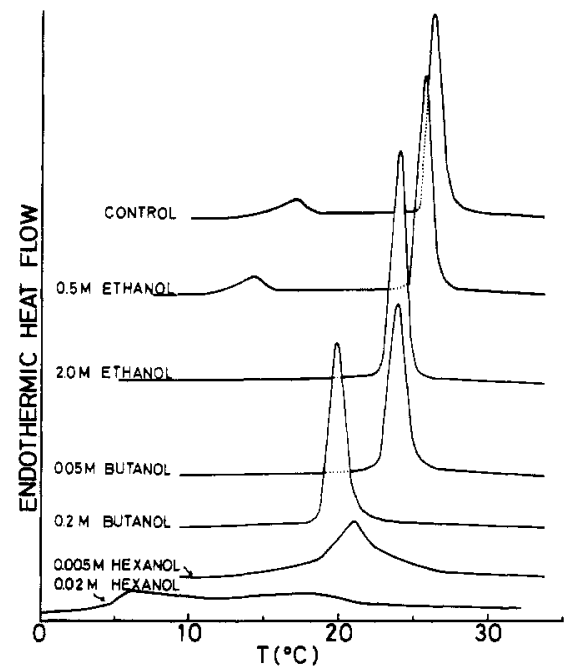

FIGURE 2: Differential scanning calorimetry of DMPC-alcohol mixtures. Alcohol concentrations (M) in lipid suspensions are indicated for each curve. The heating rate was $2.5 \mathrm{~K} \mathrm{~min}^{-1}$ at the sensitivity range of $2 \mathrm{mcal} \mathrm{s}^{-1}$.

cohols to arrive at some understanding of the effect of the alcohols at a structural level. The results are summarized in Table I. In the absence of alcohols, at $4^{\circ} \mathrm{C}$, two reflections are seen in the wide-angle region (the so-called short spacings). This is characteristic of the $L_{\beta^{\prime}}$ phase and is attributed to a 
tilt of the chain long axis with respect to the normal to the membrane plane (Tardieu et al., 1972; Janiak et al., 1976) and a consequent distorted hexagonal chain-packing lattice. In the presence of all three alcohols, there is only one reflection in the wide-angle region which indicates an erection of the chain long axis. The lattice constant is also slightly larger than in the absence of the alcohols. We also observed that the long spacing (reflections in the low-angle region) is increased in the presence of the alcohols relatively to its value in their absence. The increase in the long spacing in the presence of ethanol and 1-butanol can be accounted for simply by an erection of the chains assuming a tilt angle of about $20^{\circ}$. This is, however, not the only reason for the increase in the long spacing observed with 1-hexanol. If we assume that there is no change in the thickness of the aqueous layer in the lamellae in the presence of $0.02 \mathrm{M} 1$-hexanol, the additional $9 \AA$ can only be accounted for in a model in which 1-hexanol partitions in the midplane of the $\mathrm{L}_{\beta^{\prime}}$ phase DMPC bilayer. The results at $35^{\circ} \mathrm{C}$ are not as dramatic as in the $\mathrm{L}_{\beta^{\prime}}$ phase, but an increase in the bilayer thickness in the presence of all three alcohols may be inferred. It must be admitted, however, that the high concentration of ethanol $(2.0 \mathrm{M})$ may significantly affect the dielectric constant of the aqueous phase so that it cannot be ruled out, in this case, that there is also a contribution from an increase in the aqueous layer thickness to the overall increase in the long spacing. X-ray diffraction results with SR total lipid suspensions (results not presented) showed a less clear picture due to the difficulty of obtaining clearly multilamellar structures with this lipid mixture.

Additionally, Figure 1D shows that alcohol potency in bilayer fluidization increases with the aliphatic chain length. The ranges of added concentrations are decreased by 1 order of magnitude as the chain length increases by two carbon atoms, since bilayer/buffer partition of alcohols accordingly increase by a factor of 10 . Thus, the lipid/buffer partition coefficients for these alcohols on erythrocyte membranes (Seeman, 1972) are about $0.14,1.5$, and 13.0 for ethanol, 1-butanol, and 1hexanol, respectively. Katz and Diamond (1974) reported partitions, at $25^{\circ} \mathrm{C}$, of 0.4 and 3.2 for ethanol and 1-butanol, respectively, on DMPC/water systems, and Jain et al. (1978) reported 25.2 for hexanol on DPPC/water systems. Therefore, the concentration ranges used in our studies are within reasonable limits to compare the effects of alcohols under normalized conditions. It turns out that identical concentrations interacting with the bilayer are achieved when the added concentrations increased about 10 -fold by shortening the aliphatic chain by two carbon atoms. According to other workers, the disordering potency of short-chain primary alcohols increases with chain length and lipid solubility (Paterson et al., 1972; Zavoico \& Kutchai, 1980; Lyon et al., 1981), suggesting that the effect reflects the partition coefficient into the membrane bilayer. Also, our results indicate that, regardless of membrane partition, the fluidizing effect significantly depends on the alcohol chain length. As it increases, bilayer organization is accordingly perturbed, reflecting that bilayer geometry is modified by the length and shape of perturbing molecules, in addition to concentration.

Moreover, the perturbation type differs for the three alcohols (Figures 1 and 2), as indicated by the shapes of transition profiles reflecting the cooperativity of the phase transition (Rowe, 1983; Jain \& Wu, 1977). The characteristic sharpness of the phase transition indicates highly cooperative interactions among the lipid molecules. Ethanol and butanol, over the entire range of used concentrations, do not modify the shape and sharpness of the fluidity transition profiles (Figure 1) or

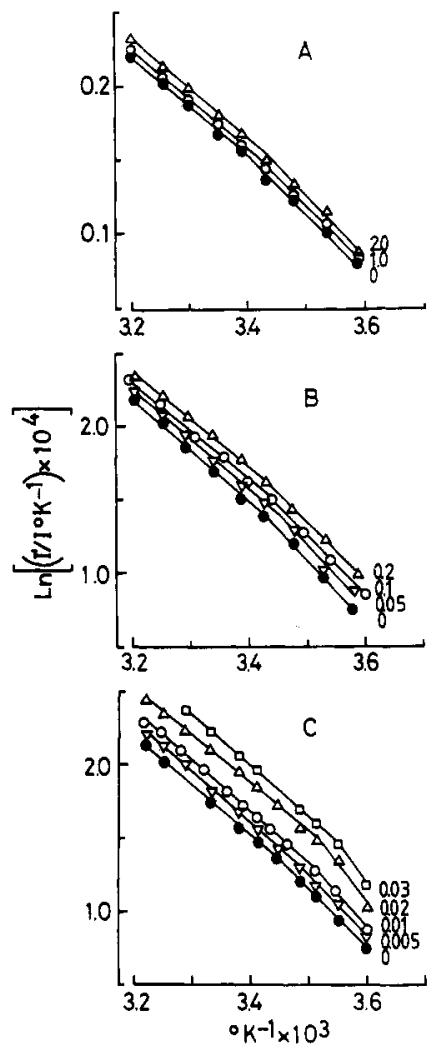

FIGURE 3: Excimer to monomer fluorescence intensity ratio, $I^{\prime} / I$, as a function of temperature for Py(3)Py in SR membranes treated with primary alcohols. (A) Ethanol; (B) 1-butanol; (C) 1-hexanol. The numbers indicate the concentration of the alcohol $(\mathrm{M})$ in membrane suspensions.

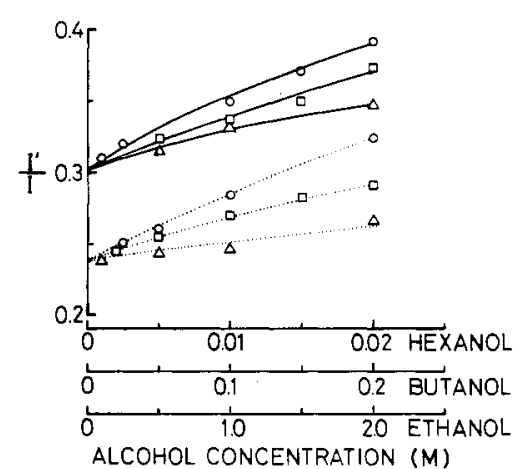

FIGURE 4: Relationship between fluorescence intensity ratio, $I^{\prime} / I$, and the added alcohol concentration in liposomes of SR lipids and in SR membranes, at $35^{\circ} \mathrm{C}$. ( $\Delta$ ) Ethanol; (ㅁ) 1-butanol; (O) 1-hexanol. Full traces refer to the results in liposomes of SR lipids and dotted traces to the results in SR membranes.

the calorimetric transitions of DMPC (Figure 2), in agreement with Jain and Wu (1977) for DPPC; also, Rowe (1983), by means of spectrophotometric measurements, reported identical results for the effect of ethanol in several phosphatidylcholine species. Conversely, 1-hexanol broadens the transition profile (Figure 1C), implying that the size of the cooperative unit undergoing transition decreases with hexanol concentration, according to the findings by means of differential scanning calorimetry (Figure 2). Again, our results agree well with those of Jain et al. (1975) and Jain and Wu (1977) for DPPC.

(b) Sarcoplasmic Reticulum Membranes. Native SR membranes and bilayers reconstituted with the total lipid extract of SR remain in the fluid state, over the temperature range from 0 to $40{ }^{\circ} \mathrm{C}$ (Almeida et al., 1982). In both membrane systems, the alcohols under study enhance the excimer formation over the entire temperature range (Figure 
3 ); this effect depends on alcohol concentration and chain length. The effects are well discernible in Figure 4 which summarizes normalized values of $I^{\prime} / I$ obtained at $35^{\circ} \mathrm{C}$. As above for DMPC, the higher fluidization potency can be assigned to hexanol, indicating that chain length, in addition to lipid solubility, determines the degree of fluidizing effect. Since each point in Figure 4 refers to identical intramembrane concentrations for the three alcohols under study, it is concluded that the chain length is an important parameter contributing for the fluidizing effect of alcohols.

Ethanol has a comparatively small fluidizing effect on SR membranes. Ethanol has been shown to increase the fluidity of biological membranes (Waring et al., 1981; Rubin \& Rottenberg, 1982; Chin \& Goldstein, 1981), but the effect depends on membrane lipid composition and depth (Chin \& Goldstein, 1981, 1984). Chin and Goldstein (1977) found that appreciable disordering of spin-labeled erythrocyte and brain membranes occurs at low aqueous concentration of ethanol (11-44 mM) in contrast with our results for SR membranes. The discrepancy is probably a consequence of the different methods used and/or due to different membrane locations of Py(3)Py and EPR probes. Nevertheless, regardless of the absolute effects, our results are still adequate for comparative purposes about the relative fluidizing effects of the alcohols under study.

Arrhenius plots of the Py(3)Py excimer formation in SR membranes consistently display a slight discontinuity at about $20^{\circ} \mathrm{C}$ related with a structural change in the lipid moiety of the membrane (Almeida et al., 1982). Whereas ethanol and butanol do not significantly affect the break position, hexanol displaces it to lower temperatures (Figure 3 ). Therefore, hexanol seems to interact differently with SR membranes similarly as described above for DMPC bilayers. A favored hexanol partition at the lipid-protein interfaces could, in principle, explain the effect. If occurring, the effect would result in perturbation (loosening) of lipid-protein interactions which would likely be reflected in functional parameters of $\mathrm{Ca}^{2+}$ pump as we shall discuss.

Effect of Alcohols on Uncoupled ATPase Activity of Sarcoplasmic Reticulum. Hydrolysis of ATP catalyzed by the $\mathrm{Ca}^{2+}$-ATPase from native vesicles of SR increases during the transport of $\mathrm{Ca}^{2+}$, until it reaches a low steady-state value when most of the added $\mathrm{Ca}^{2+}$ has been stored inside the vesicles (Weber et al., 1966; Hasselbach, 1964). The pumping process coupled to $\mathrm{Ca}^{2+}$ accumulation is only observed under conditions that allow the formation of a transmembrane gradient of $\mathrm{Ca}^{2+}$ concentration. $\mathrm{Ca}^{2+}$ ionophores and detergents uncouple the process by preventing the accumulation of $\mathrm{Ca}^{2+}$, whereas the ATP hydrolytic activity at high rates persists until all the added ATP is hydrolyzed (Carvalho \& Madeira, 1974).

Figure 5 shows the effect of ethanol, butanol, and hexanol on the uncoupled $\mathrm{Ca}^{2+}$-ATPase activity at $20^{\circ} \mathrm{C}$. Ethanol decreases the ATP-splitting activity over the entire range of concentrations used, whereas hexanol and butanol, below 0.015 and $0.15 \mathrm{M}$, respectively, enhance the activity; however, above these concentrations, a drop in activity is experienced by the ATPase. It should be emphasized that the inhibitory effects observed for ethanol are not related to enzyme denaturation since no effect of incubation time (up to $60 \mathrm{~min}$ ) was noticed. Interesting are the findings that the effects of hexanol and butanol are clearly different (Figure 5). Thus, butanol has a significant inhibitory effect at membrane concentrations which induce only minor changes in the case of hexanol. The latter only induces significant inhibition at extreme high concentrations in the membrane.

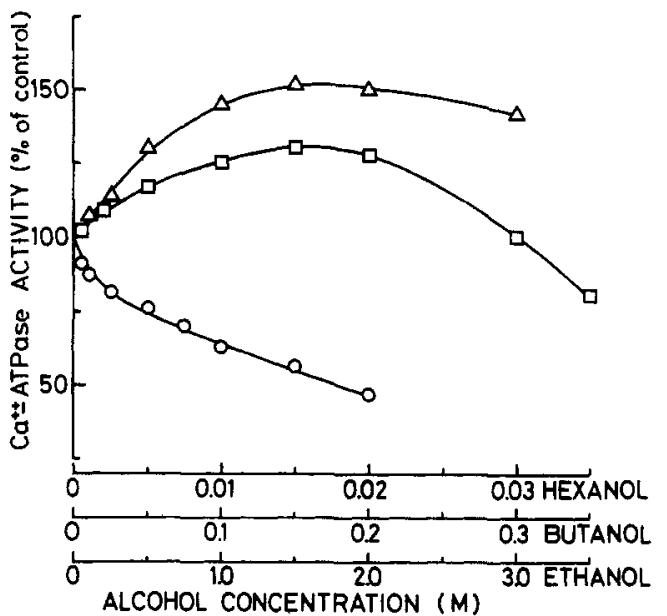

FIGURE 5: Activity of uncoupled ATP-splitting activity of sarcoplasmic reticulum as affected by different concentrations of alcohols. The activities were determined at $20^{\circ} \mathrm{C}$ in $2 \mathrm{~mL}$ of media $(50 \mathrm{mM} \mathrm{KCl}$, $5 \mathrm{mM} \mathrm{MgCl}$, and $5 \mathrm{mM}$ Tris, $\mathrm{pH}$ 6.9) containing $40 \mu \mathrm{M} \mathrm{CaCl}_{2}, 25$ $\mu \mathrm{M}$ of ionophore lasalocid (formerly X-537A), and $0.27 \mathrm{mg}$ of membrane protein. Alcohols were added directly to the final reaction mixtures in a thermostated vessel under continuous stirring, and the reactions were started by adding $1 \mathrm{mM} \mathrm{MgATP}$, after 2 min of preincubation. Activities were monitored by recording the proton production due to ATP hydrolysis. The results are expressed as percent of controls of SR incubated in similar conditions in the absence of alcohols (100\%). (O) Ethanol; (口) 1-butanol; ( $\Delta$ ) 1-hexanol.

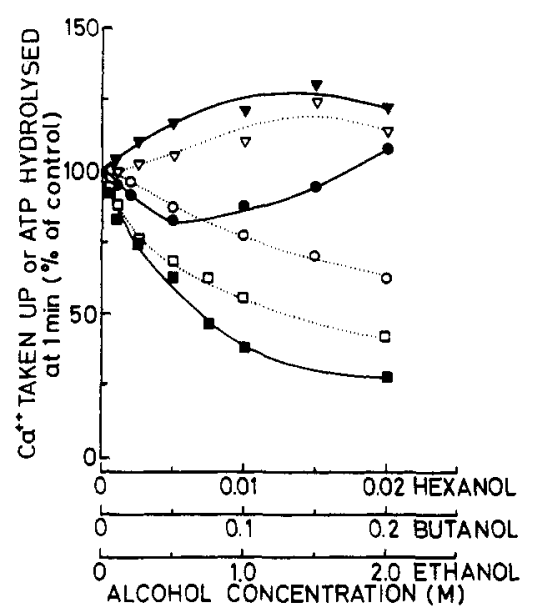

FIGURE 6: Effect of primary alcohols on $\mathrm{Ca}^{2+}$ uptake and coupled ATP hydrolysis in SR membranes. The different concentrations of alcohols were added to the final mixtures, and the reactions were monitored at $20^{\circ} \mathrm{C}$, after 2 min of preincubation. $\mathrm{Ca}^{2+}$ uptake was monitored by the use of a $\mathrm{Ca}^{2+}$ electrode, and the hydrolysis of ATP was monitored by following the production of protons with a $\mathrm{pH}$ electrode as indicated under Materials and Methods. Data represent normalized values of $\mathrm{Ca}^{2+}$ taken up and ATP hydrolyzed at $1 \mathrm{~min}$ after starting the reactions. Full symbols, ATP hydrolysis; open symbols, $\mathrm{Ca}^{2+}$ accumulation. (口) Ethanol; (O) 1-butanol; ( $\nabla$ ) 1hexanol.

Effect of Alcohols on Ca ${ }^{2+}$.Pump Efficiency. Data on the effects of ethanol, butanol, and hexanol on $\mathrm{Ca}^{2+}$ uptake and ATP hydrolysis are summarized in Figure 6. Ethanol leads to a decrease of $\mathrm{Ca}^{2+}$ uptake and ATP hydrolysis in contrast with hexanol which leads to an increase of both activities of $\mathrm{Ca}^{2+}$ pump. Butanol mimics to some extent the effect of ethanol, but above $50 \mathrm{mM}$, a stimulation of ATP hydrolysis is induced without corresponding activation of $\mathrm{Ca}^{2+}$ accumulation. Conversely to the proposal of Kondo and Kasai (1973), the decrease of $\mathrm{Ca}^{2+}$ accumulation induced by ethanol is not related with membrane leakage, since it closely parallels the ATP-splitting activity of the pump. Actually, ATP hydrolysis coupled to the translocation of $\mathrm{Ca}^{2+}$ is controlled by 
Table II: $\mathrm{Ca}^{2+} /$ ATP (Molar Ratio) As Affected by Primary Alcohols

\begin{tabular}{|c|c|c|c|c|c|}
\hline \multicolumn{2}{|c|}{ ethanol } & \multicolumn{2}{|c|}{ 1-butanol } & \multicolumn{2}{|c|}{ 1-hexanol } \\
\hline $\mathbf{M}$ & $\mathrm{Ca}^{2+} / \mathrm{ATP}$ & $\mathrm{M}$ & $\mathrm{Ca}^{2+} / \mathrm{ATP}$ & M & $\mathrm{Ca}^{2+} / \mathrm{ATP}$ \\
\hline 0 & 1.53 & 0 & 1.55 & 0 & 1.67 \\
\hline 0.05 & 1.54 & 0.01 & 1.60 & 0.0005 & 1.69 \\
\hline 0.10 & 1.58 & 0.02 & 1.63 & 0.0010 & 1.61 \\
\hline 0.25 & 1.49 & 0.05 & 1.63 & 0.0025 & 1.58 \\
\hline 0.50 & 1.61 & 0.10 & 1.38 & 0.0050 & 1.52 \\
\hline 0.75 & 2.00 & 0.15 & 1.16 & 0.0100 & 1.51 \\
\hline 1.00 & 2.22 & 0.20 & 0.90 & 0.0150 & 1.56 \\
\hline 2.00 & 2.23 & & & 0.0200 & 1.63 \\
\hline
\end{tabular}

the transmembrane $\mathrm{Ca}^{2+}$ gradient (Carvalho \& Madeira, 1974). Therefore, membrane leakiness would result in uncoupling by releasing the ATP hydrolysis from the control exerted by the transmembrane ion gradient. Rather, the energetic efficiency of $\mathrm{Ca}^{2+}$ pumping in terms of $\mathrm{Ca}^{2+} / \mathrm{ATP}$ (Table II) is maintained or even increased at high concentrations of ethanol. However, the values of $\mathrm{Ca}^{2+} / \mathrm{ATP}$ at concentrations higher than $0.5 \mathrm{M}$ may contain significant calculation errors, as a consequence of the low activities measured in these conditions.

The effect of hexanol is markedly opposed to that of ethanol. Thus, the activities of $\mathrm{Ca}^{2+}$ pump are both stimulated, indicating that the effect is exerted on the pumping rate; nevertheless, the energetic efficiency of $\mathrm{Ca}^{2+}$ pumping ( $\left.\mathrm{Ca}^{2+} / \mathrm{ATP}\right)$ is not appreciably modified (Table II). Again, our results do not support the Kondo and Kasai (1973) conclusion on leaking effects. The only agreement refers to the action of butanol for concentrations above $50 \mathrm{mM}$; accordingly, butanol uncouples the pump system by inducing membrane leaks, as suggested by the decreased efficiency $\left(\mathrm{Ca}^{2+} / \mathrm{ATP}\right)$ of $\mathrm{Ca}^{2+}$ pumping (Table II).

The discrepancy between our results and those of Kondo and Kasai (1973) is probably related with the methodologies and conditions used. We followed the kinetics of $\mathrm{Ca}^{2+}$ uptake and ATP hydrolysis by direct methods using electrometric recording systems. However, Kondo and Kasai (1973) determined $\mathrm{Ca}^{2+}$ uptake by Millipore filtration. This technique is subjected to potential artifacts in the presence of membrane active agents as described for diethyl ether (Salama \& Scarpa, 1980). These agents can alter the trapping characteristics of filters in addition to artifacts of increased membrane damage produced by pressure gradients and deformations during filtration (Salama \& Scarpa, 1980). Furthermore, by using filtration, we consistently obtain depressed $\mathrm{Ca}^{2+}$ accumulation (about half), as compared with direct measurements with the $\mathrm{Ca}^{2+}$ electrode (not shown).

Effects of Alcohols on the Ca ${ }^{2+}$-Pump Activity in the Presence of Oxalate. The above studies were substantiated by determining the effect of uptake and coupled ATPase activity in the presence of the $\mathrm{Ca}^{2+}$-trapping agent oxalate. Intravesicular $\mathrm{Ca}^{2+}$ is then osmotically inactivated, preventing it from recycling and also avoiding depression of the pump activity imposed by building up of transmembrane $\mathrm{Ca}^{2+}$ gradients. Consequently, $\mathrm{Ca}^{2+}$ uptake virtually proceeds until all the added $\mathrm{Ca}^{2+}$ is taken up. Simultaneous ATP hydrolysis proceeds at high rates until maximal $\mathrm{Ca}^{2+}$ loading of the vesicles is attained, at which time the rate of ATP hydrolysis approaches zero (Almeida et al., 1984). The effects of alcohols on $\mathrm{Ca}^{2+}$-pump activity, as expressed by ATP hydrolysis, are shown in Figure 7. Ethanol considerably extends the time interval required for the uptake of $\mathrm{Ca}^{2+}$. However, the amount of ATP hydrolyzed at the onset of the steady state is only slightly affected, thus reflecting the maintenance of pump
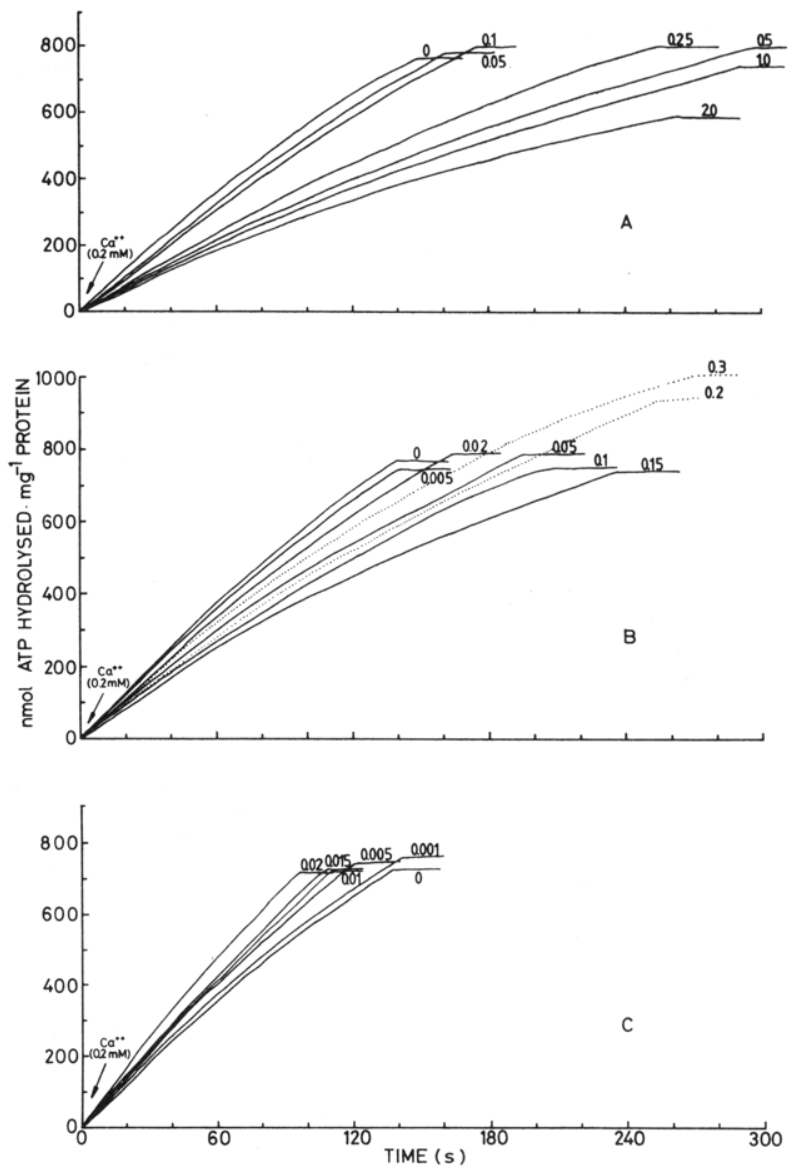

FIGURE 7: Effect of primary alcohols on the $\mathrm{Ca}^{2+}$-pump activity in the presence of oxalate. (A) Ethanol; (B) 1-butanol; (C) 1-hexanol. The activities were determined at $20^{\circ} \mathrm{C}$ in media containing $50 \mathrm{mM}$ $\mathrm{KCl}, 5 \mathrm{mM} \mathrm{MgCl}, 5 \mathrm{mM}$ Tris, $\mathrm{pH} 6.9,5 \mathrm{mM}$ potassium oxalate, $0.5 \mathrm{mM} \mathrm{MgATP}$, and $0.27 \mathrm{mg}$ of SR protein. The reactions were started by adding $0.2 \mathrm{mM} \mathrm{CaCl}_{2} 2 \mathrm{~min}$ after addition of alcohol. The hydrolysis of ATP was monitored by following the production of protons with a $\mathrm{pH}$ electrode. Alcohol concentrations (M) in lipid suspensions are indicated for each curve.

efficiency $\left(\mathrm{Ca}^{2+} / \mathrm{ATP}\right)$. As described above for studies in the absence of oxalate, ethanol only decreases the rate of $\mathrm{Ca}^{2+}$ pumping without affecting its efficiency.

Ethanol affects DMPC lipid bilayer organization as detected by $\mathrm{Py}(3) \mathrm{Py}$, calorimetry, and X-ray diffraction, but small effects were detected in SR membranes at concentrations up to $0.5 \mathrm{M}$. Yet, at these concentrations, ethanol markedly inhibits the coupled and uncoupled $\mathrm{Ca}^{2+}$-pump activities. Since SR lipid structure organization is not appreciably affected, it is tempting to conclude that ethanol interacts superficially with the membrane, affecting either lipid-protein polar interactions or interacting directly with the polar extrinsic moiety of the $\mathrm{Ca}^{2+}$-ATPase enzyme which contains the active sites for ATP and $\mathrm{Ca}^{2+}$ interaction. Dielectric constant alterations at the active site by ethanol presence may also be involved in depression of the $\mathrm{Ca}^{2+}$-pump rate. This effect could be mediated by interference with hydration and dehydration steps occurring during the $\mathrm{Ca}^{2+}$ translocation cycle (Dupont, 1983).

Similarly to ethanol, butanol up to $0.15 \mathrm{M}$ also extends the time interval required for the uptake of $\mathrm{Ca}^{2+}$ while the energetic efficiency of $\mathrm{Ca}^{2+}$ pumping $\left(\mathrm{Ca}^{2+} / \mathrm{ATP}\right.$ ratio) is maintained (Figure 7B). Above $0.15 \mathrm{M}$, the inhibition of the $\mathrm{Ca}^{2+}$ pump is associated with a decrease of energetic efficiency which reflects partial uncoupling, as a consequence of membrane leakiness, as previously suggested (Kondo \& Kasai, 1973; Hara \& Kasai, 1977). Our studies in the absence of oxalate (Figure 6 and Table II) provide similar qualitative 
evidence, although the uncoupling effect is already detected for butanol concentrations above $0.05 \mathrm{M}$. The $\mathrm{Ca}^{2+}$-sequestering action of oxalate effectively aborts development of large $\mathrm{Ca}^{2+}$ gradients across the membrane, thus opposing efflux of $\mathrm{Ca}^{2+}$ in conditions of partial leakiness induced by moderate concentrations of butanol. However, at higher concentrations, oxalate cannot compensate for the extensive membrane damage induced by butanol. Lenaz et al. $(1975,1978)$ have claimed that butanol affects ATPase activity and properties in mitochondria through the perturbation of lipid bilayer environment of membrane proteins; however, Vanderkooi et al. (1981) pointed out that those effects may as well be related to direct interaction with membrane proteins rather than with lipids. Garda and Brenner (1984) have also provided evidence that the activity of some microsomal membrane-bound enzymes can be accelerated by an increase of membrane fluidity induced by butanol. Our results cannot overrule the possibility that some of the effects of butanol on membranes are mediated in part by the increase in membrane fluidity, but a clear correlation between membrane fluidity and SR activity cannot be established.

Conversely to ethanol and butanol, hexanol stimulates the $\mathrm{Ca}^{2+}$-pump rate, since the time required for the uptake of $\mathrm{Ca}^{2+}$ is decreased (Figure 7C); nevertheless, the $\mathrm{Ca}^{2+} / \mathrm{ATP}$ ratio is not significantly affected, as in the case of oxalate absence. As concluded from the described physical studies on DMPC bilayers and SR membranes, hexanol presumably interacts deeply as compared with more superficial interactions of the other alcohols. Additionally, hexanol perturbs the bilayer organization in a different way, interfering with the cooperative association of lipid molecules (Figures 1 and 2) and increasing the bilayer thickness significantly (Table I). Moreover, its fluidizing potency is remarkable either in DMPC or in SR membranes (Figures 1, 3, and 4). On the other hand, hexanol is the only alcohol that specifically stimulates the $\mathrm{Ca}^{2+}$ pump, strongly suggesting that this effect is mediated through alterations of physical properties occurring in the lipid phase or at lipid-protein interfaces. Therefore, we suggest that modulation of membrane fluidity by hexanol regulates the $\mathrm{Ca}^{2+}$-pump activity presumably by affecting the conformational flexibility of the $\mathrm{Ca}^{2+}$-pump system.

Previous conclusions of East et al. (1984) that membrane fluidity does not regulate the $\mathrm{Ca}^{2+}$-ATPase of SR are faulty. The ATPase of SR has been reconstituted into phospholipid bilayers of different fatty acyl chain length. Therefore, the bilayer fluidity has been changed simultaneously with the membrane thickness. The effect of bilayer thickness on the ATPase activity (Johannsson et al., 1981) was not taken into account by East et al. (1984). Therefore, their conclusions about the effect of fluidity are premature. We have already shown (Almeida et al., 1984) that the decrease in membrane fluidity induced by incorporation of cholesterol is likely to impose physical constraints upon the ionophoretic pathway of the $\mathrm{SR} \mathrm{Ca}^{2+}$-pump system, decreasing its activity. Therefore, the opposite effect resulting from membrane fluidization induced by hexanol strongly supports the hypothesis that membrane fluidity modulates the activity of the $\mathrm{Ca}^{2+}$-pump system. Enzyme inhibition (uncoupled activity) observed at high hexanol concentrations is presumably related with lipid withdrawal from direct contact with the enzyme; lipid to ATPase effective ratio may, thus, decrease below the critical value of about 30-40 (Hesketh et al., 1976; Benett et al., 1980).

\section{ConClusions}

Ethanol, 1-butanol, and 1-hexanol perturb the bilayer or- ganization in model membranes of DMPC and SR lipids or in native SR membranes as detected by intramolecular excimer formation of incorporated Py(3)Py, calorimetry, and X-ray diffraction. The alcohols have a fluidizing effect on membranes, but their potency depends on concentration in lipid suspension and on aliphatic chain length. Additionally, hexanol extensively perturbs the lipid organization as revealed by the alteration of phase transition cooperativity in DMPC bilayers and of the lipid bilayer thickness.

The different effects of the alcohols on the activity of SR membranes ruled out a unitary mechanism of action on the basis of fluidity changes. Whereas ethanol inhibits the $\mathrm{Ca}^{2+}$ pumping, hexanol stimulates it. Nevertheless, the energetic efficiency of $\mathrm{Ca}^{2+}$ pumping ( $\mathrm{Ca}^{2+} / \mathrm{ATP}$ ), regardless the $\mathrm{ab}$ solute pumping rates, is maintained. Similarly to ethanol, butanol also decreases the $\mathrm{Ca}^{2+}$-pumping rate over a limited range of added concentrations. However, the energetic efficiency of $\mathrm{Ca}^{2+}$ pumping is decreased at higher concentrations. Depending on the chain length, the alcohols interact with the SR membranes in different regions, perturbing differently the $\mathrm{Ca}^{2+}$-pump enzyme. Apparently, some of the effects of ethanol and butanol may be related to alteration of lipid-protein polar interactions or to direct interaction with the polar moiety of the $\mathrm{Ca}^{2+}$-ATPase itself. Presumably, the effect of hexanol is mainly exerted through alterations of physical properties occurring in the lipid phase or lipid-protein interfaces. These alterations modulate the $\mathrm{Ca}^{2+}$-pump activity.

Registry No. DMPC, 13699-48-4; ATP, 56-65-5; ATPase, 9000-83-3; Ca, 7440-70-2; EtOH, 64-17-5; BuOH, 71-36-3; HO$\left(\mathrm{CH}_{2}\right)_{5} \mathrm{Me}, 111-27-3$.

\section{REFERENCES}

Almeida, L. M., Vaz, W. L. C., Zachariasse, K. A., \& Madeira, V. M. C. (1982) Biochemistry 21, 5972-5977.

Almeida, L. M., Vaz, W. L. C., Zachariasse, K. A., \& Madeira, V. M. C. (1984) Biochemistry 23, 4714-4720.

Bartlett, G. R. (1959) J. Biol. Chem. 234, 466-468.

Benett, J. P., McGill, K. A., \& Warren, G. B. (1980) Curr. Top. Membr. Transp. 14, 127-164.

Böttcher, C. J. F., van Gent, C. M., \& Pries, C. (1961) Anal. Chim. Acta 24, 203-204.

Carvalho, A. P., \& Madeira, V. M. C. (1974) in Biomembranes-Lipids, Proteins and Receptors (Burton, R. M., \& Packer, L., Eds.) pp 347-367, BI-Science Publication Division, Webster Groves, MO.

Chin, J. H., \& Goldstein, D. B. (1977) Mol. Pharmacol. 13, 435-441.

Chin, J. H., \& Goldstein, D. B. (1981) Mol. Pharmacol. 19, 425-431.

Chin, J. H., \& Goldstein, D. B. (1984) Lipids 19, 929-935. Dupont, Y. (1983) FEBS Lett. 161, 14-20.

East, J. M., Jones, O. T., Simmonds, A. C., \& Lee, A. G. (1984) J. Biol. Chem. 259, 8070-8071.

Franks, N. P., \& Lieb, W. R. (1978) Nature (London) 274 , 339-342.

Garda, H. A., \& Brenner, R. R. (1984) Biochim. Biophys. Acta 769, 160-170.

Gornall, A. G., Bardawill, C. J., \& David, M. M. (1949) J. Biol. Chem. 177, 751-766.

Hara, K., \& Kasai, M. (1977) J. Biochem. (Tokyo) 82, 1005-1017.

Hasselbach, W. (1964) Prog. Biophys. 14, 167-222.

Hasselbach, W. (1979) Top. Curr. Chem. 78, 1-56.

Haydon, D. A., Hendry, B. M., \& Levinson, S. R. (1977) Nature (London) 268, 356-358. 
Hesketh, T. R., Smith, G. A., Houslay, M. D., McGill, K. A., Birdsall, N. J. N., Metcalfe, J. C., \& Warren, G. B. (1976) Biochemistry 15, 4145-4151.

Hill, M. W. (1974) Biochim. Biophys. Acta 356, 117-124.

Houslay, M. D., \& Stanley, K. K. (1982) in Dynamics of Biological Membranes, Wiley, New York.

Jain, M. K., \& Wu, N. Y.-M. (1977) J. Membr. Biol. 34, 157-201.

Jain, M. K., Wu, N. Y.-M., \& Wray, L. V. (1975) Nature (London) 255, 494-495.

Jain, M. K., Gleeson, J., Upreti, A., \& Upreti, G. C. (1978) Biochim. Biophys. Acta 509, 1-8.

Janiak, M. J., Small, D. M., \& Shipley, G. G. (1976) Biochemistry 15, 4575-4580.

Johannsson, A., Keightley, C. A., Smith, G. A., \& Metcalfe, J. C. (1981) Biochem. J. 196, 505-511.

Katz, Y., \& Diamond, J. M. (1974) J. Membr. Biol. 17, 101-120.

Kondo, M., \& Kasai, M. (1973) Biochim. Biophys. Acta 311, 391-399.

Lee, A. G. (1976) Biochemistry 15, 2448-2454.

Lenaz, G., Parenti-Castelli, G., \& Sechi, A. M. (1975) Arch. Biochem. Biophys. 167, 72-79.

Lenaz, G., Bertoli, E., Curatola, G., Mazzanti, L., \& Bigi, A. (1976) Arch. Biochem. Biophys. 172, 278-288.

Lenaz, G., Curatola, G., Mazzanti, L., Parenti-Castelli, G., \& Bertoli, E. (1978) Biochem. Pharmacol. 27, 2835-2844.

Lyon, R. C., \& Goldstein, D. B. (1983) Mol. Pharmacol. 23, 86-91.

Lyon, R. C., McComb, J. A., Schreurs, J., \& Goldstein, D. B. (1981) J. Pharmacol. Exp. Ther. 218, 669-675.

Madeira, V. M. C. (1975) Biochem. Biophys. Res. Commun. 64, 870-876.

Madeira, V. M. C. (1978) Arch. Biochem. Biophys. 185, 316-326.

Madeira, V. M. C. (1982) Cell Calcium 3, 67-79.

Madeira, V. M. C., \& Antunes-Madeira, M. C. (1976) Cienc. Biol. (Coimbra) 2, 265-291.

Melnick, R. L., Haspel, H. C., Goldenberg, M., Greenbaum, L. M., \& Weinstein, S. (1981) Biophys. J. 34, 499-515.

Pang, K.-Y. Y., Braswell, L. M., Chang, L., Sommer, T. J., \& Miller, K. W. (1980) Mol. Pharmacol. 18, 84-90.
Paterson, S. J., Butler, K. W., Huang, P., Labelle, J., Smith, I. C. P., \& Schneider, H. (1972) Biochim. Biophys. Acta 266, 597-602.

Retig, J. N., Kirchberger, M. A., Rubin, E., \& Katz, A. M. (1977) Biochem. Pharmacol. 26, 393-396.

Richards, C. D., Martin, K., Gregory, S., Keightley, C. A., Hesketh, T. R., Smith, G. A., Warren, G. B., \& Metcalfe, J. C. (1978) Nature (London) 276, 775-779.

Rowe, E. S. (1983) Biochemistry 22, 3299-3305.

Rubin, E., \& Rottenberg, H. (1982) Fed. Proc., Fed. Am. Soc. Exp. Biol. 41, 2465-2471.

Salama, G., \& Scarpa, A. (1980) J. Biol. Chem. 255, 6525-6528.

Seeman, P. (1972) Pharmacol. Rev. 24, 583-655.

Stokes, J. A., \& Harris, R. A. (1982) Mol. Pharmacol. 22, 99-104.

Stümpel, J., Vaz, W. L. C., \& Hallmann, D. (1985) Biochim. Biophys. Acta 821, 165-168.

Sturtevant, J. M. (1982) Proc. Natl. Acad. Sci. U.S.A. 79, 3963-3967.

Swartz, M. H., Repke, D. I., Katz, A. M., \& Rubin, E. (1974) Biochem. Pharmacol. 23, 2369-2376.

Tada, M., Yamamoto, T., \& Tonomura, J. (1978) Physiol. Rev. 58, 1-79.

Tardieu, A., Luzzati, V., \& Reman, F. C. (1972) J. Mol. Biol. 75, 711-733.

Trudell, J. R., Hubell, W. L., \& Cohen, E. N. (1973a) Biochim. Biophys. Acta 291, 321-327.

Trudell, J. R., Hubell, W. L., \& Cohen, E. N. (1973b) Biochim. Biophys. Acta 291, 328-334.

Vanderkooi, G., Shaw, J., Storms, C., Vennerstrom, R., \& Chignell, D. (1981) Biochim. Biophys. Acta 635, 200-203.

Waring, A. J., Rottenberg, H., Ohnishi, T., \& Rubin, E. (1981) Proc. Natl. Acad. Sci. U.S.A. 78, 2582-2586.

Weber, A., Herz, R., \& Reiss, I. (1966) Biochem. Z. 345, 329-369.

Zachariasse, K. A., Kühnle, W., \& Weller, A. (1980) Chem. Phys. Lett. 73, 6-11.

Zachariasse, K. A., Vaz, W. L. C., Sotomayor, C., \& Kühnle, W. (1982) Biochim. Biophys. Acta 688, 323-332.

Zavoico, G. B., \& Kutchai, H. (1980) Biochim. Biophys. Acta $600,263-269$. 